\title{
Thermal model development and validation for rapid filling of high pressure hydrogen tanks
}

\author{
Terry Johnson ${ }^{1}$, Radoslav Bozinoski ${ }^{2, *}$, Jianjun $\mathrm{Ye}^{3}$, George Sartor ${ }^{1}$, \\ Jinyang Zheng ${ }^{3}$, Jian Yang ${ }^{3}$
}

\section{Abstract}

This paper describes the development of thermal models for the filling of high pressure hydrogen tanks with experimental validation. Two models are presented; the first uses a one-dimensional, transient, network flow analysis code developed at Sandia National Labs, and the second uses the commercially available CFD analysis tool Fluent. These models were developed to help assess the safety of Type IV high pressure hydrogen tanks during the filling process. The primary concern for these tanks is due to the increased susceptibility to fatigue failure of the liner caused by the fill process. Therefore, a thorough understanding of temperature changes of the hydrogen gas and the heat transfer to the tank walls is essential. The effects of initial pressure, filling time, and fill procedure were investigated to quantify the temperature change and verify the accuracy of the models. In this paper we show that the predictions of mass averaged gas temperature for the one

\footnotetext{
${ }^{*}$ Corresponding author

Email address: rbozino@sandia.gov (Radoslav Bozinoski)

${ }^{1}$ Transportation Energy center, Sandia National Laboratories, Livermore, CA, USA

${ }^{2}$ Thermal/Fluid Science \& Engineering Dept., Sandia National Laboratories, Livermore, CA, USA

${ }^{3}$ Institute of Process Equipment, Zhejiang University, Hangzhou, Zhejiang, P. R. China
} 
and three-dimensional models compare well with the experiment and both can be used to make predictions for final mass delivery. Due to buoyancy and other three-dimensional effects, however, the maximum wall temperature cannot be predicted using one-dimensional tools alone which means that a three-dimensional analysis is required for a safety assessment of the system. Keywords:

High pressure hydrogen storage, fast filling, real gas equation, one-dimensional and 3D models

\section{Introduction}

Hydrogen is widely regarded as an energy carrier that could help to overcome issues related to greenhouse gas emissions, air pollution and energy security $[1,2]$. The use of hydrogen as a fuel for vehicles requires a safe and optimized method to store a sufficient amount of hydrogen. Therefore, hydrogen storage is one of the most important problems for hydrogen applications $[3,4,5]$. Currently, the most acceptable method for storing hydrogen on vehicles is by high pressure tanks. This is due to their practicality, reliability, and durability. At present there are two main types of tanks used: Type III, comprised of a carbon-fiber/epoxy composite external wrap with an internal aluminum liner; and Type IV, with the same external composite wrap but an internal plastic liner $[6,7]$.

Due to the low volumetric energy density of hydrogen gas, high pressure storage is needed to compete with gasoline-fueled vehicles. For widespread adoption of hydrogen-powered vehicles, refueling time should also be comparable with that of conventional fueling (3-5 minutes for a passenger vehicle). 
Therefore, rapid filling is combined with high storage pressures in order to meet competitive targets for short refueling times, high refueling rates, and long driving ranges. Fast refueling of hydrogen gas, however, induces a significant temperature rise in the tanks due to the compression effect[8]. This temperature rise creates two potential problems: 1) the gas heats the tank liner which can jeopardize the structural integrity of the tank; 2) the high temperature reduces the hydrogen density in the tank which reduces the final mass delivery. This, in turn, reduces the driving range of the hydrogen vehicles. Therefore, the temperature increase during filling represents a safety and efficiency concern. For safety reasons, international regulation ISO 15869 [9] specifies a maximum allowed temperature of $85^{\circ} \mathrm{C}$ during filling of hydrogen tanks. Therefore, a thorough understanding of the fill process is necessary to meet the standards and safety regulations.

Previous articles have focused on the temperature rise during fast filling of high pressure hydrogen tanks. Winters et. al. have developed a onedimensional tank model to predict the time varying pressure and temperature of compressed gas in a tank as it is being filled [10]. Monde et. al. have also built a one-dimensional model to study the temperature rise during the fast filling processes [11]. Their results show good agreement between experimental and predicted results for the gas temperature rise during the filling process.

Liu and Zhao have also developed a two-dimensional CFD model for the fast filling of $70 \mathrm{MPa}$ hydrogen vehicle tanks [4]. Their model is based on an axisymmetric algorithm that neglects buoyancy effects. Their numerical results slightly under-predict the experimental results. 
Dicken and Merida have conducted elaborate experimental and numerical studies on the temperature distribution during hydrogen refueling. In their experiments a $74 \mathrm{~L}$ type III hydrogen tanks was internally instrumented with 63 thermocouples distributed along the middle vertical plane. Effects of filling time and initial mass on the temperature rise were investigated [12]. They also proposed a numerical model based on symmetric flow where the effects of gravitational and buoyancy forces were ignored. However, the temperature estimated by their model seems to be in poor agreement with the measured values.

Heitsch[13] et. al. simulated the fast filling of hydrogen tanks using the commercial CFD software CFX. They presented two studies on the influence of the initial pressure of the gas and liner material to the increase in gas temperature in the tank.

Suryan[14] et. al. have developed a three-dimensional computational model. They incorporated real gas effects to more accurately predict the thermo-fluid dynamic behavior of hydrogen gas during filling of a type III high pressure tank. In their three-dimensional analysis, the real gas behavior is captured by the Redlich-Kwong model and the effects of ambient, initial, and inlet gas temperatures are studied. By incorporating three-dimensional effects and real-gas behavior they were able to achieve numerical results that were within a maximum difference of $2.5^{\circ} \mathrm{C}$ from experiment. Kim and Lee have conducted a series of experiments and 3D CFD simulations to quantify the temperature change of a tank during the fast fill process [15]. The results show the gas temperature in the upper part of the cylinder was higher than that of the lower part due to buoyancy effect. They also found that the 
difference in temperature between the upper and lower part of the tank was higher for their CFD predictions than what was measured from experiment.

Galassi has presented a 3D CFD model for hydrogen fast filling[16]. The simulation of hydrogen fast filling at different working conditions were performed and the accuracy of the numerical models was assessed against experimental data for a type IV tank up to $70 \mathrm{MPa}$. Predicted temperatures inside the tank were found to be in very good agreement with experimental data for both test cases and the errors on maximum calculated temperatures did not exceed $7 \%$. Nevertheless, the evaluation of external tank and front boss temperatures were less accurate (errors above 10\%) and were shown to be strongly dependent on material properties.

The above previous articles show that 3D models have better accuracy compared to one-dimensional and two-dimensional models. The tradeoff is that the 3D model will use more mesh cells and CPU time. In this paper, one-dimensional and 3D models are built for comparison. The numerical modeling validation against experimental data at different working conditions will be presented. The effect of the fill condition on the thermal behavior of the tank will be analyzed and discussed.

\section{Fast Filling Experiments}

All of the experiments were conducted in a hydrogen fueling lab with high pressure storage units at Sandia National Laboratories (SNL). These fast fill tests were performed using a 36.9 liter type IV tank, with a $3.8 \mathrm{~mm}$-thick plastic liner, and a 23.5mm-thick wrapping of carbon-fiber reinforced epoxy composite. The tank has a length of $905 \mathrm{~mm}$ and $319 \mathrm{~mm}$ diameter, as shown 


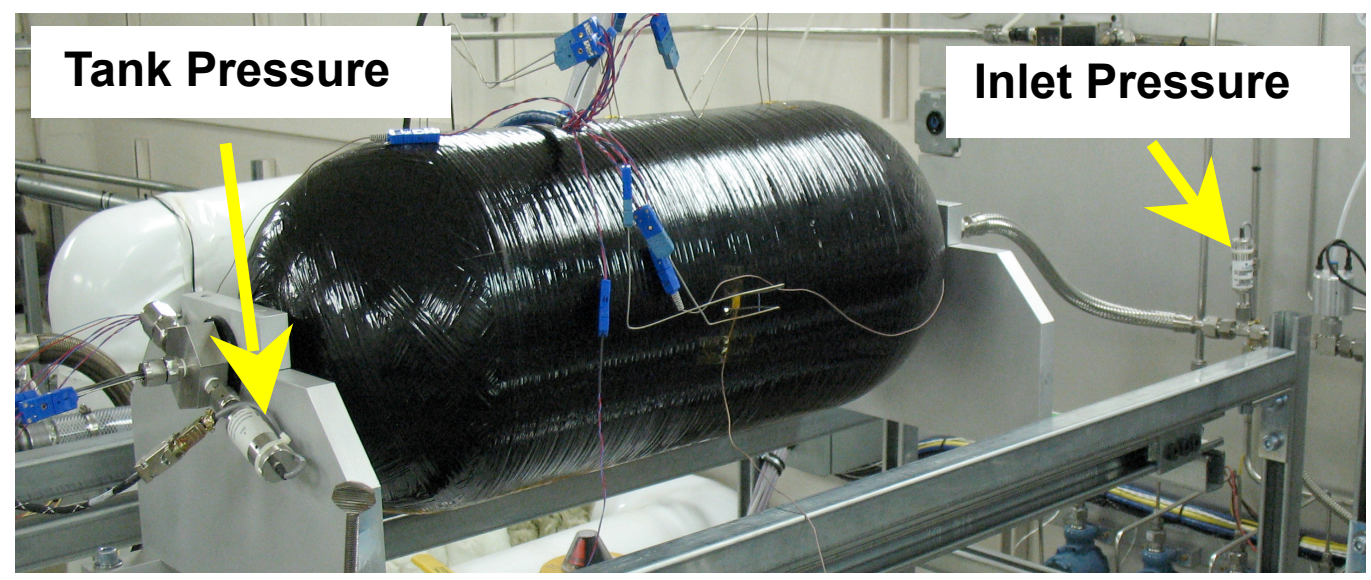

Figure 1: Filling test system

in Figure 1. In order to measure the temperature rise of the hydrogen gas and the tank during the fueling processes, 22 thermocouples were arranged as depicted in Figure 2.

There are five thermocouples ${ }^{1}$ (1-5) spaced along the tank length to detect the hydrogen gas temperature in the pressure vessel. The remaining locations (6-9) each contain four thermocouples that are located at the inner liner surface of the tank, at the liner and carbon fiber reinforced polymer (CFRP) interface, in the middle of the CFRP layer, and at the outer surface of the tank. The final thermocouple was located upstream of the tank inlet and provides a measurement of the incoming hydrogen gas temperature $^{2}$. The mounting fixture allowed the tank to be rotated such that the

\footnotetext{
${ }^{1}$ It should be noted that these thermo couples were fed into the tank through the inlet using stiff support wires. These held the sensors at the nominal axial centerline location but due to gravity, each successive sensor would sag a little more than the previous.

${ }^{2}$ note shown in Figure 2
} 


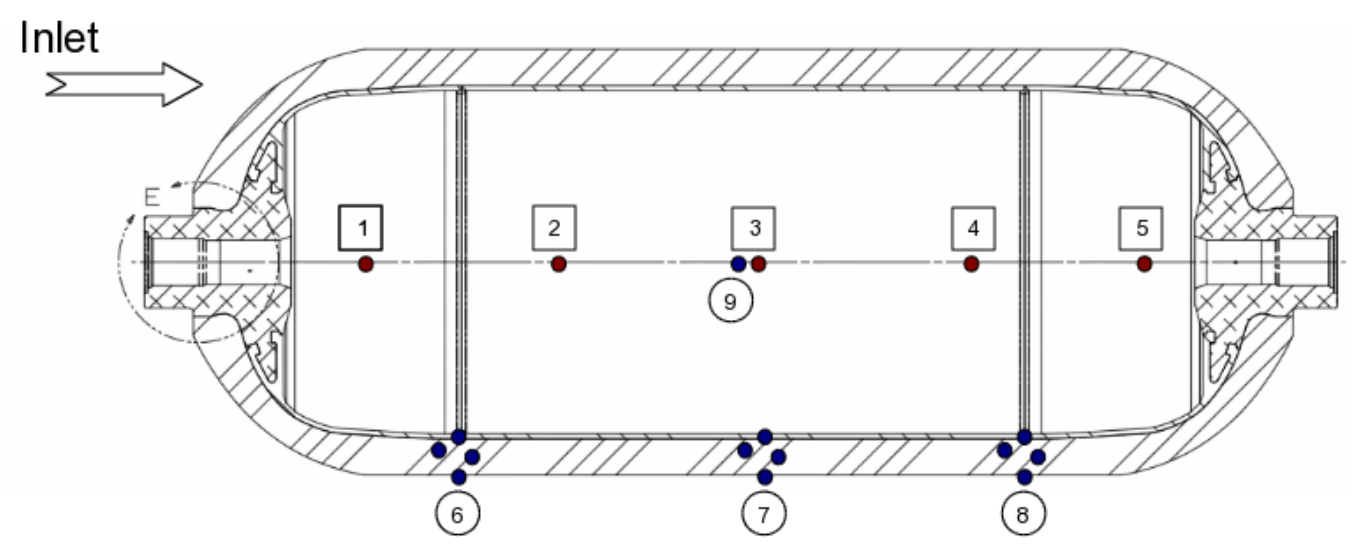

Figure 2: Position of the thermocouples

thermocouples could be positioned at different angular locations. The first set of experiments was conducted with the three axially distributed sets of thermocouples (locations 6-8) at 12 o'clock and the fourth (location 9) set at 3 o'clock, and will be referred to experimental orientation 1. A number of these experiments were then repeated after the tank was rotated 180 degrees so that the three sets (locations 6-8) were at 6 o'clock and the fourth set (location 9) was at 9 o'clock, and will be referred to as experimental orientation 2. The gas pressure was measured using pressure transducers located just upstream of the tank inlet and is shown in Figure 2. All of the experiments were conducted using linear pressure ramps and used an automated pressure regulator. The mass flowrate was recorded using a Coriolis mass flow meter.

Several hydrogen fueling processes were implemented for the fast filling tests. Prior to initiating each test, the tank pressure was brought to the desired starting pressure and the tank temperature was allowed to equili- 
Table 1: Conditions of the fast fill experiments

\begin{tabular}{|c|c|c|c|c|c|}
\hline Filling test & $\begin{array}{l}\text { Initial } \\
\text { Pressure }\end{array}$ & $\begin{array}{c}\text { Final } \\
\text { Pressure }\end{array}$ & $\begin{array}{l}\text { Filling } \\
\text { Times }\end{array}$ & $\begin{array}{c}\text { Initial } \\
\text { Temperature }\end{array}$ & $\begin{array}{l}\text { Avg. Inlet } \\
\text { Temperature }\end{array}$ \\
\hline & $(\mathrm{MPa})$ & $(\mathrm{MPa})$ & $(\mathrm{s})$ & $(\mathrm{C})$ & $(\mathrm{C})$ \\
\hline Case 1 & 0.14 & 13.79 & 45 & 23.5 & 23.4 \\
\hline Case 2 & 0.17 & 13.79 & 90 & 23.2 & 24.9 \\
\hline Case 3 & 0.14 & 13.79 & 90 (three steps) & 24.8 & 28.8 \\
\hline Case 4 & 2.02 & 13.79 & 90 & 24.9 & 20.5 \\
\hline Case 5 & 4.99 & 13.79 & 90 & 27.9 & 20.3 \\
\hline
\end{tabular}

brate $^{1}$. In this study, various initial pressures, fill times, final pressures, and fill procedures were investigated. Table 1 presents the parameters for five of these tests which will be discussed in more detail. The initial gas pressure, final gas pressure, filling time, environment temperature, average inlet gas temperature, and the amount of the charged hydrogen gas are specified for each test.

\section{Formulation \& Solution Methodology}

\subsection{D numerical model}

A simple one-dimensional transient model for tank filling was developed using the network flow analysis code Netflow. Netflow models compressible flow in networks consisting of pressure vessels, connecting tubing, orifices, valves and flow branches $[10,17]$. One-dimensional transient heat conduction

\footnotetext{
${ }^{1}$ The temperature of the room was held at $23^{\circ} \mathrm{C}$ for all the experiments.
} 
in the vessel or tube walls can also be modeled. For the convenience of the reader, some features of the Netflow model are presented here.

Normally, a tank or vessel would be modeled in Netflow as a single control volume. In this case a the tank is modeled as a connected series of three control volumes each with unique wall geometry. A schematic of the tank represented by Netflow is shown in Figure 3.

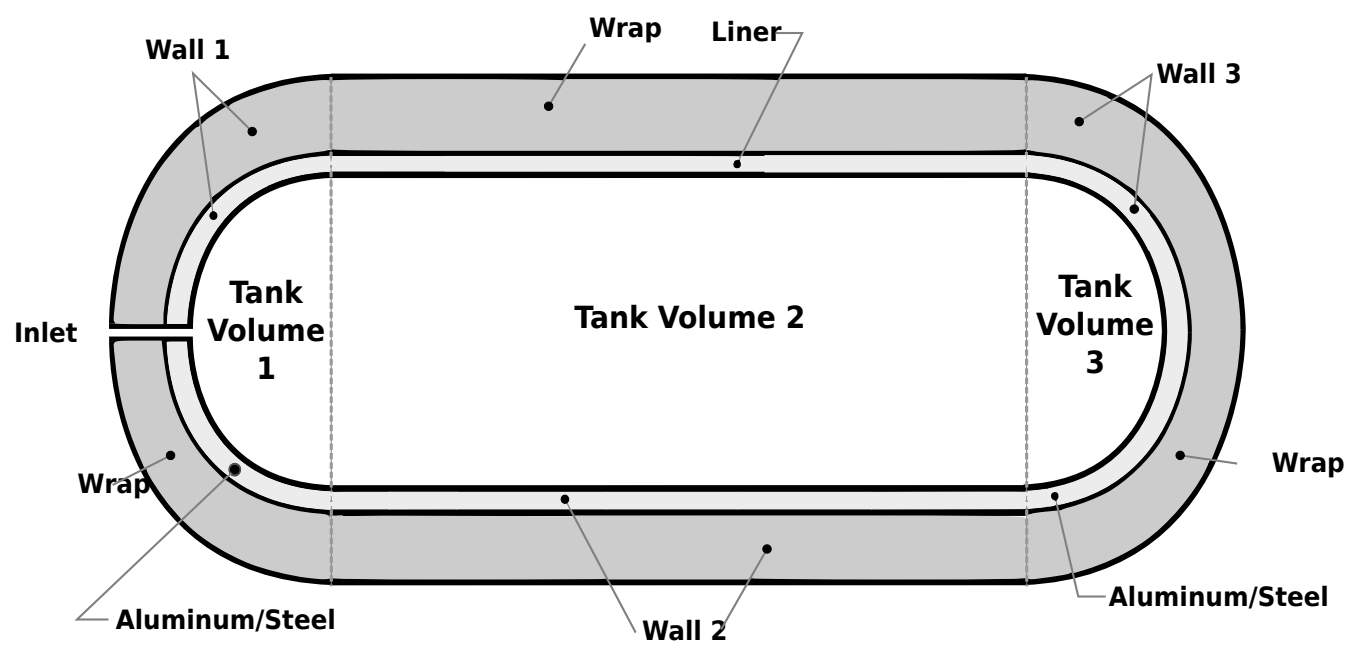

Figure 3: one-dimensional Tank Model

The largest portion of the tank (control volume 2 and wall 2) is represented by a cylinder. The inlet side of the tank (control volume 1 and wall 1) and the back of the tank (control volume 3 and wall 3) are modeled as half spheres. The sum of volumes 1,2 , and 3 is equal to the actual tank volume. The wall thicknesses of the liner and wrap in wall 2 are identical to those found in the cylindrical portion of the tank. Some compromises had to be made in modeling the wrap and liner in walls 1 and 3 since the layer 
thicknesses were not uniform in these locations. Furthermore, the tank walls in these locations contain a combination of structural aluminum and steel surrounded by a wrap. An effort was made to assign layer thicknesses and material properties in such a way as to approximate the actual heat capacity and heat conduction path in the tank walls.

Mass conservation and energy for the gas in each control volume were modeled using Equations 1 and 2

$$
\begin{gathered}
\frac{d(\rho \forall)}{d t}=\dot{m}_{i}-\dot{m}_{e} \\
\frac{d(\rho \forall u)}{d t}=\dot{m}_{i}\left(H+\frac{1}{2} v^{2}\right)_{i}-\dot{m}_{e}\left(H+\frac{1}{2} v^{2}\right)_{e}-h A_{s}\left(T-T_{w i}\right)
\end{gathered}
$$

where $\dot{m}, \rho, u, H, T, v, \forall, A_{s}, T_{w i}$ and $h$ are the mass flow rate, gas density, gas internal energy, gas enthalpy, gas temperature, gas velocity, gas volume, interior tank wall surface area, interior tank wall temperature and the heat transfer coefficient. The subscripts $i$ and $e$ refer to inlet and exit flows for each control volume. For volume 3(far side of the tank from the inlet) the middle term in Equation 2 does not exist. Except for the mass flow at the tank inlet, all the kinetic energy terms are negligible. Netflow computes the gas internal energy and enthalpy as $C_{v} T$ and $C_{p} T$ with constant specific heats.

The three control volume model will produce unique gas and wall temperatures in each section of the tank. Since pressure drop between the control volumes is negligible, the pressure computed for the gas will be uniform throughout the tank. 
Since the fill pressure in the tank exceeds ideal gas conditions, a real gas equation-of-state (EOS) is used to relate gas density, pressure and temperature. Netflow utilizes the Able-Noble form of the van der Waals EOS in which the molecular attraction constant is neglected and the co-volume constant is slightly changed [18]. The resulting EOS is

$$
P=\frac{\rho R T}{1-b \rho}
$$

where $R$ is the gas constant and $b$ is the adjusted co-volume constant. The co-volume constant determined by [18] for hydrogen $\left(7691 \frac{\mathrm{m}^{3}}{\mathrm{~kg}}\right)$ is used in the present work.

Heat is transferred from the gas at temperature, $T$, to the interior wall, $T_{w}$, according to Newtons law of cooling. The heat transfer model for the tank walls is shown schematically in Figure 4.

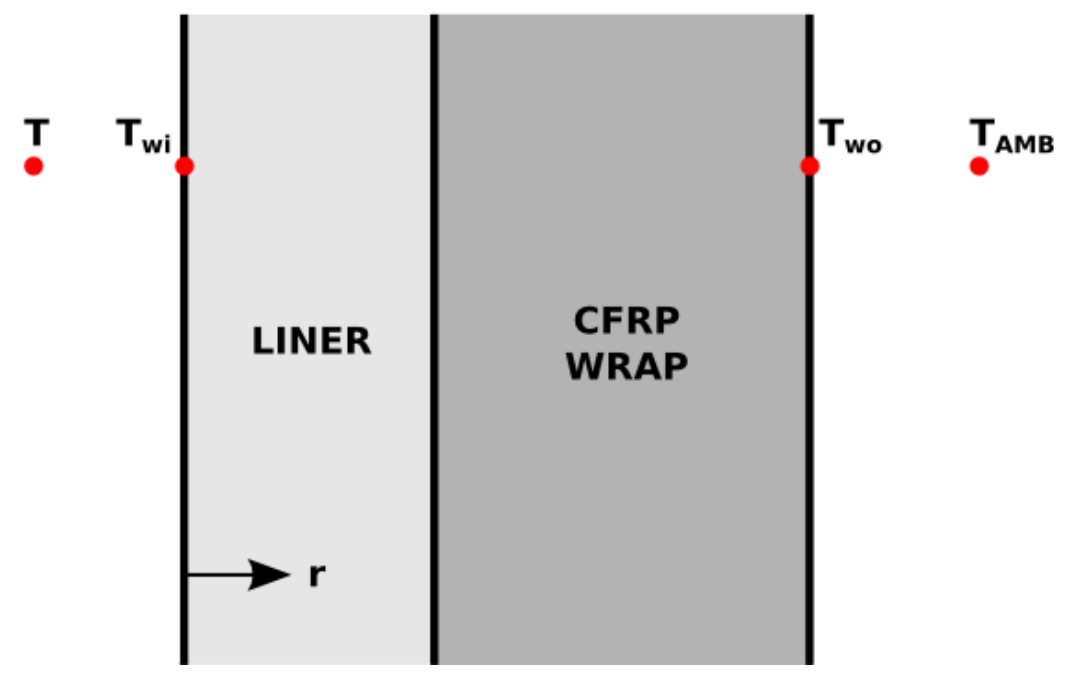

Figure 4: one-dimensional Heat Transfer Model

From the interior tank wall at temperature $T_{w_{i}}$ to the exterior tank wall 
at temperature $T_{w_{o}}$, heat is transferred via one-dimensional transient heat conduction through each layer. For walls 1 and 3, the spherical sections of the tank, the heat conduction equation takes the form

$$
\rho_{w} C_{w} \frac{d T_{w}}{d t}=\frac{k_{w}}{r^{2}} \frac{\partial}{\partial r}\left(r^{2} \frac{\partial T}{\partial r}\right)
$$

where $T_{w}, k_{w}, \rho_{w}$, and $C_{w}$ are the wall temperature, thermal conductivity, density, and specific heat respectively.

For wall 2, the cylindrical section of the tank, the heat conduction equation takes the form

$$
\rho_{w} C_{w} \frac{d T_{w}}{d t}=\frac{k_{w}}{r} \frac{\partial}{\partial r}\left(r \frac{\partial T}{\partial r}\right)
$$

Equation 4 and 5 are solved in Netflow using finite differences through the thickness $(r)$ direction. The number of divisions in each wall layer can be defined by the user. Twenty divisions per layer was more than enough to achieve grid independent solutions. The heat transfer between the exterior wall and the ambient was modeled using Newtons law of cooling, i.e.

$$
q_{a}=h_{a m b}\left(T_{w o}-T_{a m b}\right)
$$

where $q_{a}, T_{w o}, T_{a m b}$ and $h_{a m b}$ are the heat flux per unit area, outside wall temperature, ambient temperature and the heat transfer coefficient. A natural convection heat transfer coefficient of $3.65 \frac{\mathrm{W}}{\mathrm{m}^{2} \mathrm{~K}}$ was used for $h_{a m b}$. Results were relatively insensitive to the value of $h_{a m b}$. The heat transfer coefficient ( $h$ in Equation 2) used to describe the transfer of heat from the gas to the interior tank wall was determined from experiments assuming that the heat 
transfer mechanism was a combination of forced and free convection. Following the approach of others[19, 20, 21] the forced and free convection Nusselt numbers, $N u_{\text {forced }}$ and $N u_{\text {free }}$, were determined from

$$
\begin{gathered}
N u_{\text {forced }}=C_{1} R e^{C_{2}} \\
N u_{\text {free }}=C_{3} R a^{1 / 3}
\end{gathered}
$$

where the Reynolds number, $R e$, and the Rayleigh number, $R a$, are given by:

$$
\begin{gathered}
R e=\frac{\rho d v}{\mu} \\
R a=\frac{g \beta\left(T-T_{w i}\right) \rho^{2} D^{3} C p}{\mu^{2} k}
\end{gathered}
$$

The Reynolds number in Equation 9 is determined from tank inlet conditions where $\rho, v, \mu$ and $d$ are the inlet gas density, velocity, viscosity and diameter. The Rayleigh number in Equation 10 is determined from control volume conditions where $\mathrm{g}$ is the gravitational constant, $D$ is the characteristic diameter of the control volume, and $C_{p}, \beta, \mu$, and $k$ are the gas specific heat at constant pressure, volume expansivity, dynamic viscosity, and thermal conductivity respectively. The forced and free convection Nusselt numbers were combined using the method suggested by Ranong et. al. $[22]$, i.e.

$$
N u=\frac{h D}{k}=\left(h_{\text {forced }}^{4}+h_{\text {free }}^{4}\right)^{1 / 4}
$$


In Equation 8, the exponent on the Rayleigh number was set to $\frac{1}{3}$ which is typical for turbulent free convection. The constants $C_{1}, C_{2}$, and $C_{3}$ in Equations 7 and 8 were determined experimentally from the five tests documented in Table 1. The constants were modified to produce the best comparison of the experimentally measured gas mass-averaged temperature and the Netflow computed gas mass-averaged temperature. The best fit was obtained for $C_{1}=0.6, C_{2}=0.632$ and $C_{3}=.3$. The gas mass-averaged temperature for the tank is expressed mathematically by

$$
T_{\text {avg }}=\frac{\int_{\forall} T d m}{m}
$$

and represents a measure of gas energy content as a function of time. In Equation $12 T_{\text {avg }}, T, m$ and $\forall$ are the gas mass-averaged temperature, local gas temperature, gas mass and total tank volume respectively.

\subsection{D Numerical Model}

The mass conservation equation is given by:

$$
\frac{\partial \rho}{\partial t}+\frac{\partial}{\partial x_{i}}\left(\rho u_{i}\right)=0
$$

where $t$ is time, $\rho$ is gas density, $u_{i}$ the velocity tensor, and $x_{i}$ denotes the the coordinate direction. The subscript $i=1,2,3$ denotes the $x, y$, and $z$ directions, respectively.

In an inertial coordinate system the law of conservation of momentum is given by: 


$$
\begin{aligned}
\frac{\partial \rho u_{i}}{\partial t}+\frac{\partial \rho u_{i} u_{j}}{\partial x_{j}}= & -\frac{\partial P}{\partial x_{i}}+\frac{\partial}{\partial x_{j}}\left[\mu\left(\frac{\partial u_{i}}{\partial x_{j}}+\frac{\partial u_{j}}{\partial x_{i}}-\frac{2}{3} \delta_{i j}\right)\right] \\
& -\frac{\partial}{\partial x_{j}}\left(\rho \overline{u_{i}^{\prime} u_{j}^{\prime}}\right)+F_{Y}
\end{aligned}
$$

where $P$ is the static pressure, $F_{Y}$ is the gravitational body force and is given by $F_{Y}=\rho g_{i}$.

For Turbulence modeling, the $k-\epsilon$ model is often chosen for turbulent flows with heat transfer. In this paper a modified version of the turbulence model is adopted. The turbulence kinetic energy, $k$, and its rate of dissipation, $\epsilon$, are obtained from the following transport equations:

$$
\begin{gathered}
\frac{\partial \rho k}{\partial t}+\frac{\partial \rho k u_{i}}{\partial x_{i}}=\frac{\partial}{\partial x_{j}}\left[\left(\mu+\frac{\mu_{t}}{\sigma_{k}}\right) \frac{\partial k}{\partial x_{j}}\right]+G_{k}+G_{b}-\rho \epsilon-Y_{M} \\
\frac{\partial \rho \epsilon}{\partial t}+\frac{\partial \rho \epsilon u_{i}}{\partial x_{i}}=\frac{\partial}{\partial x_{j}}\left[\left(\mu+\frac{\mu_{t}}{\sigma_{\epsilon}}\right) \frac{\partial \epsilon}{\partial x_{j}}\right]+C_{1 \epsilon}\left(G_{k}+C_{3 \epsilon} G_{b}\right) \frac{\epsilon}{k}-C_{2 \epsilon} \rho \frac{\epsilon^{2}}{k}
\end{gathered}
$$

where $C_{1 \epsilon, 2 \epsilon, 3 \epsilon}$ are constants and $\sigma_{k, \epsilon}$ are the turbulent Prandtl numbers for $k$ and $\epsilon$, respectively. $G_{k}$ represents the generation of turbulence kinetic energy due to the mean velocity gradients, given by:

$$
G_{k}=-\rho \overline{u_{i}^{\prime} u_{j}^{\prime}} \frac{\partial u_{j}}{\partial x_{i}}
$$

$G_{b}$ is the modified buoyancy component of turbulence kinetic energy and given by:

$$
G_{b}=\beta g_{i} \frac{\mu_{t}}{P r_{t}} \frac{\partial T}{\partial x_{i}}
$$


where $\beta$ is the thermal expansion coefficient of hydrogen and defined as:

$$
\beta=-\frac{1}{\rho}\left(\frac{\rho}{\rho T}\right)_{P}
$$

$Y_{M}$ represents the contribution of the fluctuating dilatation in compressible turbulence to the overall dissipation rate and is described by:

$$
Y_{M}=2 \rho \epsilon M_{t}^{2}
$$

where $M_{t}$ is the turbulent Mach number defined as:

$$
M_{t}=\sqrt{\frac{k}{a^{2}}}
$$

with $a$ representing the speed of sound.

The turbulent viscosity, $\mu_{t}$ is calculated by the following:

$$
\mu_{t}=\rho C_{\mu} \frac{k}{\epsilon^{2}}
$$

where $C_{\mu}$ is a constant.

The conservation of energy equation is given by:

$$
\begin{gathered}
\frac{\partial \rho E}{\partial t}+\frac{\partial}{\partial x_{i}}\left(u_{i}(\rho E+P)\right)=\frac{\partial}{\partial x_{j}}\left[k_{e f f} \frac{\partial T}{\partial x_{j}}+u_{i}\left(\tau_{i j}\right)_{e f f}\right] \\
E=h-(p / \rho)+(1 / 2) \nu^{2}
\end{gathered}
$$

where $k_{\text {eff }}$ is the effective thermal conductivity defined as follows:

$$
k_{e f f}=k_{g a s}+\frac{c_{p} \mu_{t}}{P r_{t}}
$$


Here, $k_{\text {gas }}$ is the thermal conductivity of hydrogen. The effective viscosity is given by the following equation:

$$
\left(\tau_{i j}\right)_{e f f}=\mu_{e f f}\left[\frac{\partial u_{i}}{\partial x_{j}}+\frac{\partial u_{j}}{\partial x_{i}}-\frac{2}{3} \delta_{i j} \frac{\partial u_{k}}{\partial x_{k}}\right]
$$

where $\mu_{\text {eff }}=\mu+\mu_{t}$.

In the 3D simulations, the National Institute of Standards and Technology (NIST) real gas model ${ }^{1}$ is used to account for the compressibility effect of high pressure hydrogen gas. The modified Benedict-Webb-Rubin equation of state is given by NIST to describe the PVT relationship of hydrogen gas, which is presented as a function of temperature and density.

$$
P=\sum_{n=1}^{9} \alpha_{n} \rho^{n}+\exp \left[{\frac{\rho}{\rho^{c r i t}}}^{2}\right] \sum_{n=10}^{1} 5 \alpha_{n} \rho^{2 n-17}
$$

The critical density, $\rho^{\text {crit }}$, and leading coefficients, $\alpha_{n}$, are functions of temperature. In total there are 32 adjustable parameters. All of the thermodynamic properties can be found in Younglove and Mclinden [23].

For the 3D model the geometry is divided into two domains: the fluid domain filled with hydrogen gas and the solid domain that includes the liner, the composite over wrap, the aluminum bosses at either end and the inlet tube. The computational domain representing the tank was generated with the ANSYS workbench meshing software. There are approximately 250,000 cells of a hybrid type mesh which represent the fluid domain inside the tank and the solid domain of the tank. A 3D mesh of the Type IV tank is shown in Figure 5.

\footnotetext{
${ }^{1}$ REFPROP v7.0
} 


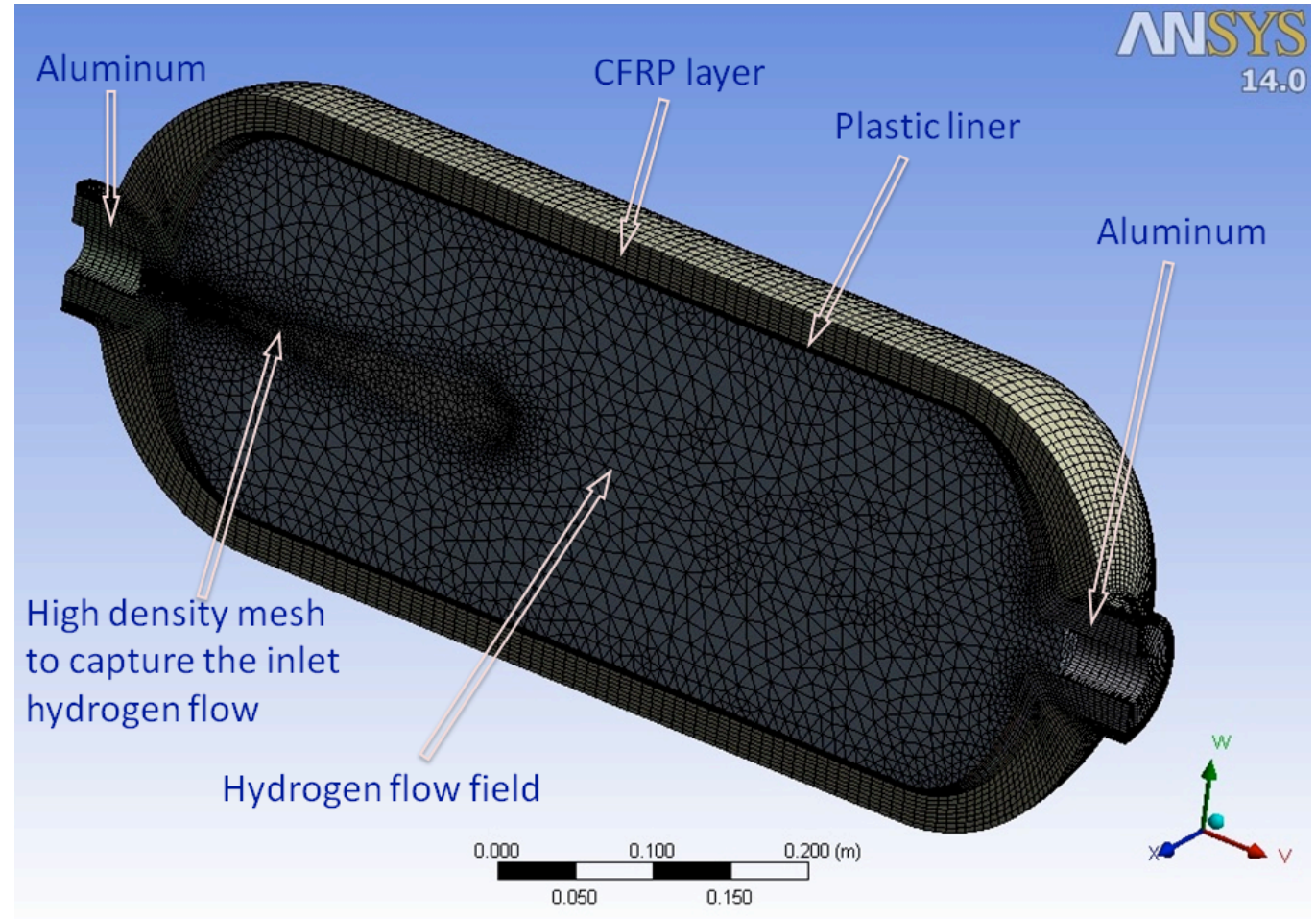

Figure 5: 3D model of type IV tank

The commercially available CFD package, ANSYS FLUENT 14.0, was used to solve the coupled set of transient equations presented earlier. Hydrogen was selected as the fluid domain gas using the Fluent database with compressibility effects modeled using the NIST thermodynamics real gas library. Viscous effects are modeled with the standard $k-\epsilon$ equations with buoyancy effects included. A pressure inlet boundary condition was specified at the tank inlet and varied with time to match experimental conditions. Inlet gas temperature was also set to the averaged inlet gas temperature of the experiment. 


\section{Results and Discussion}

Here we present the results obtained using the one-dimensional model to simulate the experiment listed as Case 5 in Table 1. Results for the other cases are similar.

Figure 6 shows a comparison of the measured and computed gas massaveraged temperature as a function of time during the tank fill.

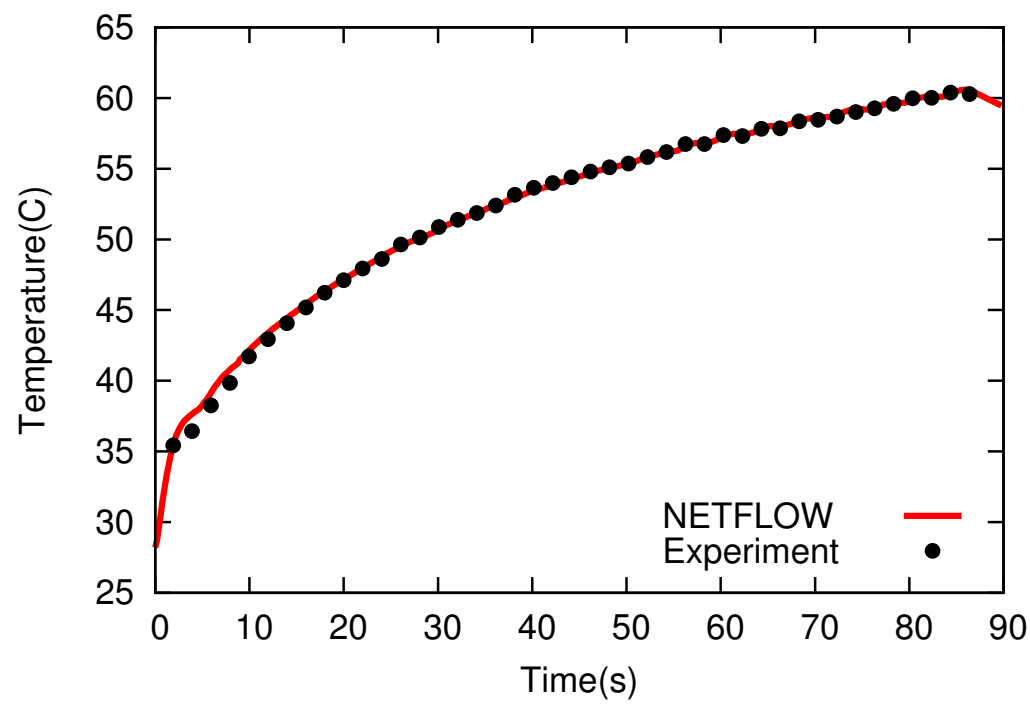

Figure 6: Comparison of Measured and Computed Gas Mass-Averaged Temperatures.

The computed control volume temperatures for volume 1, 2 and 3 were mass-averaged to produce a single temperature for direct comparison to the measurements. The Netflow simulation does an excellent job of replicating the temperature measurements. This is true not only for the gas massaveraged temperature but also the mass inventory and pressure in the tank as a function of time. 
Figure 7 compares the measured and computed interior wall temperatures at a specific location.

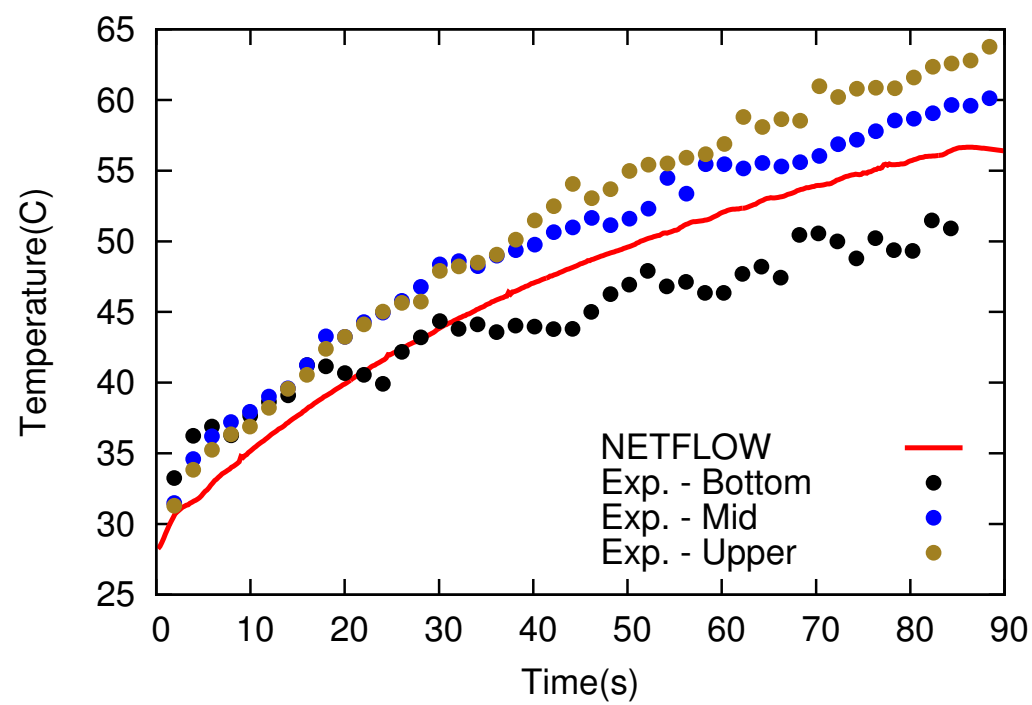

Figure 7: Comparison of Measured and Computed Interior Wall Temperatures.

The tank orientation during the fill was such that the tank axis was parallel to the ground. During the fill, natural convection in the tank causes gradients in gas such that the gas in the upper portion of the tank is hotter than the gas in the lower portion. This effect is missing in the one-dimensional model since single control volumes are used for both the top and bottom of the tank. The temperature gradients in the gas cause temperature gradients in the tank walls. This is clearly seen in Figure 7 by comparing measured temperatures for upper, midpoint and bottom interior temperature. The Netflow-computed interior wall temperature falls between the experimentally measured values. This is to be expected since the entire circumference of the 
tank wall is lumped into a single thickness ${ }^{1}$ heat conduction path. Hence, in a lower fidelity model (i.e. one-dimensional) predicted wall temperatures are likely to fall below the peak wall temperatures.

It should be noted that the first Netflow models of the tank filling process were done with a single gas control volume for the entire tank and a single heat conduction path for the tank walls. These models could be made to replicate measured mass-averaged temperature but not interior wall temperatures. Any reasonable averaging of tank wall layer thicknesses and properties produced computed interior tank wall temperatures that were far lower than any of the measured or computed transients shown in Figure 7. This underscores the importance of dealing with tank wall thickness and property variations over the tank wall. This was the principle reason for developing a three control volume model so that difference in the tank wall could be accounted for at some level.

Figure 8 shows the measured mass flow-rates for the 5 cases listed in Table 1. The initial mass, filled mass and the total storage mass for the 5 cases are given in Table 2. The mass-averaged temperature for the 5 cases are shown in Figure 9 along with the predicted mass-averaged temperature from the 3D model. The experimental mass-averaged temperature is calculated based on the measured mass flow-rate for each case using the Able-Noble hydrogen gas equation. As with the one-dimensional model, the 3D results are in good agreement with the experimental mass-averaged temperature. The maximum difference between the simulation and the experimental results is about $3^{\circ} \mathrm{C}$.

\footnotetext{
${ }^{1}$ See figure 4
} 
The mass inventory and pressure also match the experimental results. Note that the measured inlet pressure, not the mass flow rate, was used as the boundary condition for the 3D model.

Table 2: Fill tank experimental results.

\begin{tabular}{|c|c|c|c|c|c|}
\hline Case & 1 & 2 & 3 & 4 & 5 \\
\hline \hline Initial mass (g) & 5.5 & 5.3 & 5.2 & 59.8 & 143.0 \\
\hline Filled mass (g) & 307.2 & 324.7 & 323.5 & 276.1 & 196.8 \\
\hline Total storage mass (g) & 312.7 & 330.0 & 328.7 & 335.9 & 339.8 \\
\hline Maximum Gas Temperature $\left({ }^{\circ} \mathrm{C}\right)$ & 87 & 72 & 77 & 65 & 60 \\
\hline
\end{tabular}




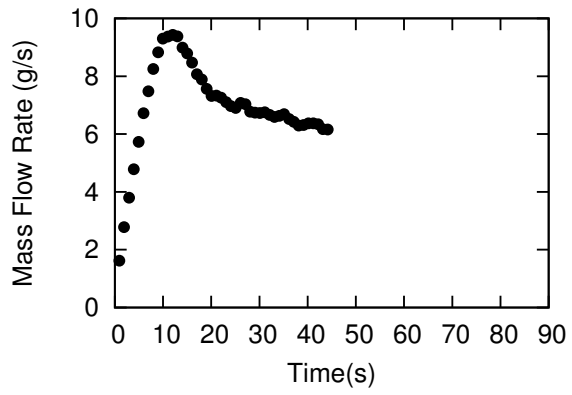

(a) case 1

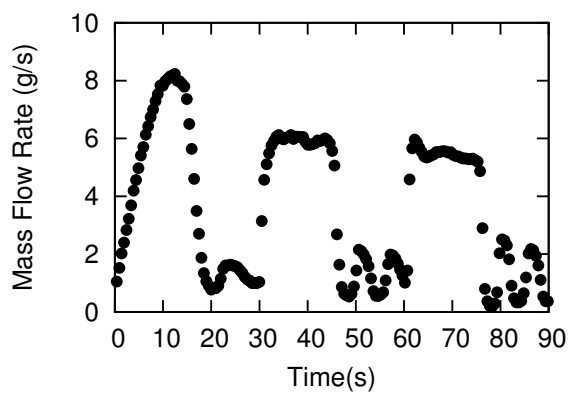

(c) case 3

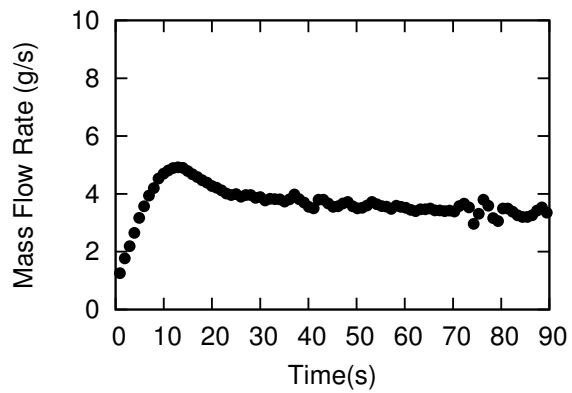

(b) case 2

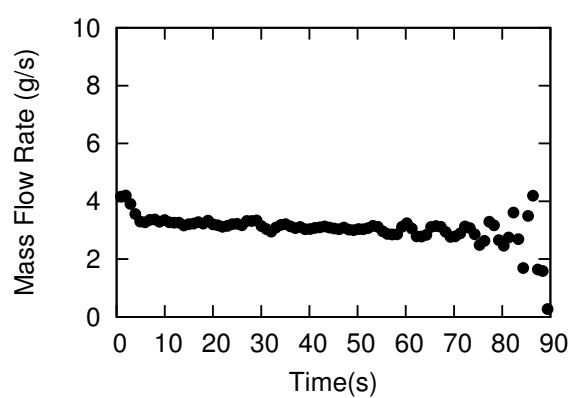

(d) case 4

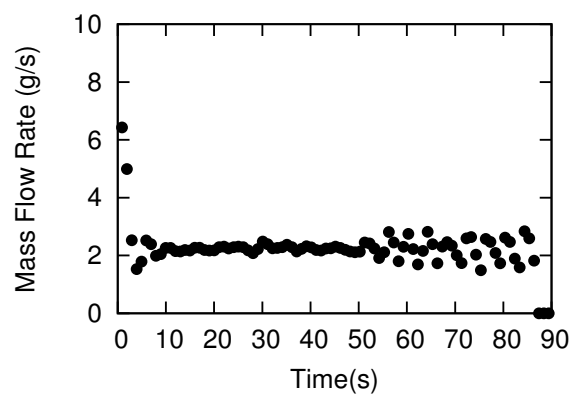

(e) case 5

Figure 8: Experimental mass flow rate 
Case 1 is the fastest of the five cases with a 45-second linear pressure ramp fill. Figure 8 shows the mass flow-rate peaks at about $9.5 \mathrm{~g} / \mathrm{sec}$ with an average of about $6.8 \mathrm{~g} / \mathrm{sec}$ over the fill. Figure 9(a) shows the gas temperature rises rapidly to $70^{\circ} \mathrm{C}$ in the beginning seconds, then the rate slows and reaches about $87^{\circ}$ at the end of the fill. Case 2 is a 90-second fill over the same pressure range as Case 1 that results in a fill rate half that of Case 1. The results show a less severe temperature spike, when compared to case 1, and a final temperature of $70^{\circ} \mathrm{C}$. This was due to the lower fill rate and longer fill time. Because of the lower temperature, and a resulting lower back pressure, Case 2 had a higher mass fill which was $5.5 \%$ greater than Case 1 .

In Case 3, the total fill time and pressure range is the same as Case 2; however, the fill has three 30-second steps shown in Figure 8. Three corresponding temperature spikes can also be seen in Figure 9(c). The highest temperature is $78^{\circ} \mathrm{C}$ and occurs at the last step. The maximum temperature is $8^{\circ} \mathrm{C}$ higher than the maximum temperature of Case 2. Table 2 shows that the mass of $H_{2}$ filled in Case 3 is about the same as in Case 2. Since the peak temperature is lower, however, the linear pressure ramp of Case 2 would be preferred.

Case 4 and Case 5 are 90-second linear fills, like Case 2, but with higher tank initial pressures at $290 \mathrm{psi}$ and $725 \mathrm{psi}$, respectively. This leads to weaker compressibility effects leading to lower temperatures and higher total storage mass. In case 4 , the highest temperature is approximately $65^{\circ} \mathrm{C}$ and the final mass is $335.9 \mathrm{~g}$, which is $7.4 \%$ more than case 1 . In case 5 , the highest temperature is approximately $60^{\circ} \mathrm{C}$ and the final mass is $339.8 \mathrm{~g}$, which is $8.7 \%$ more than case 1 . 


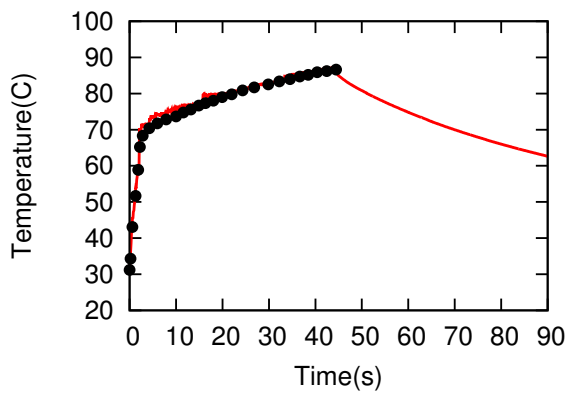

(a) case 1

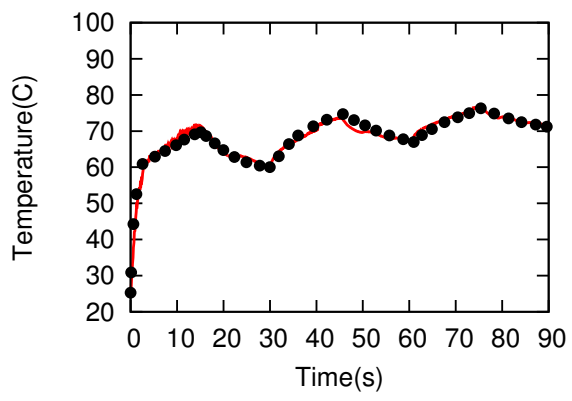

(c) case 3

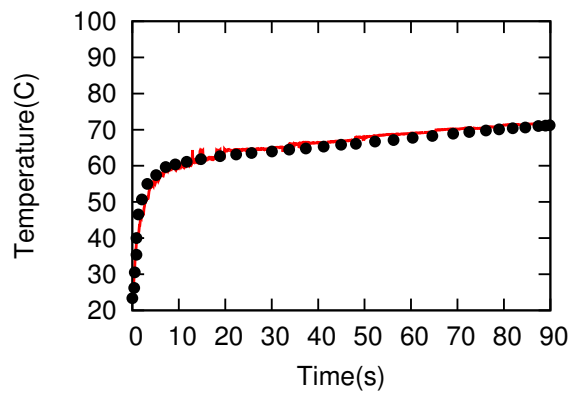

(b) case 2

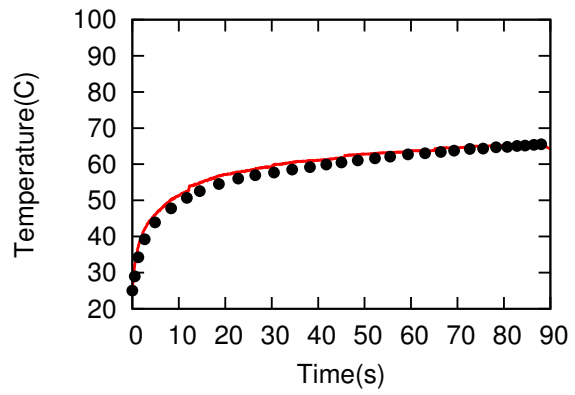

(d) case 4

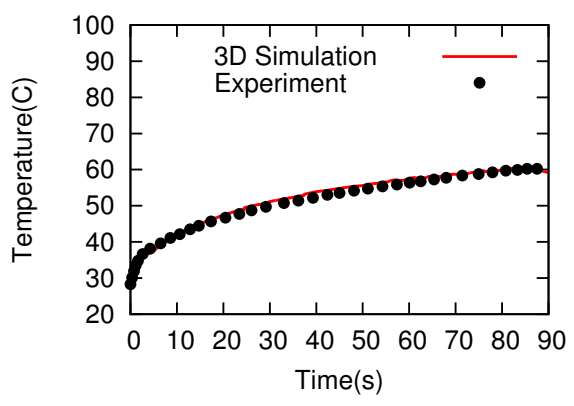

(e) case 5

Figure 9: Comparisons between the experimentally averaged temperatures and the simulation gas temperatures. 
So far we have only considered average gas temperature and mass delivery, however, from a safety standpoint the maximum temperature in the tank is of primary interest. Therefore, it is important to investigate the temperature distribution inside the tank. For this investigation we focused on a single case, Case 5. Figure 10 shows measured and predicted temperature rise envelopes of the gas. Experimental envelope bands are shown for both orientations corresponding to the description in section 2 .

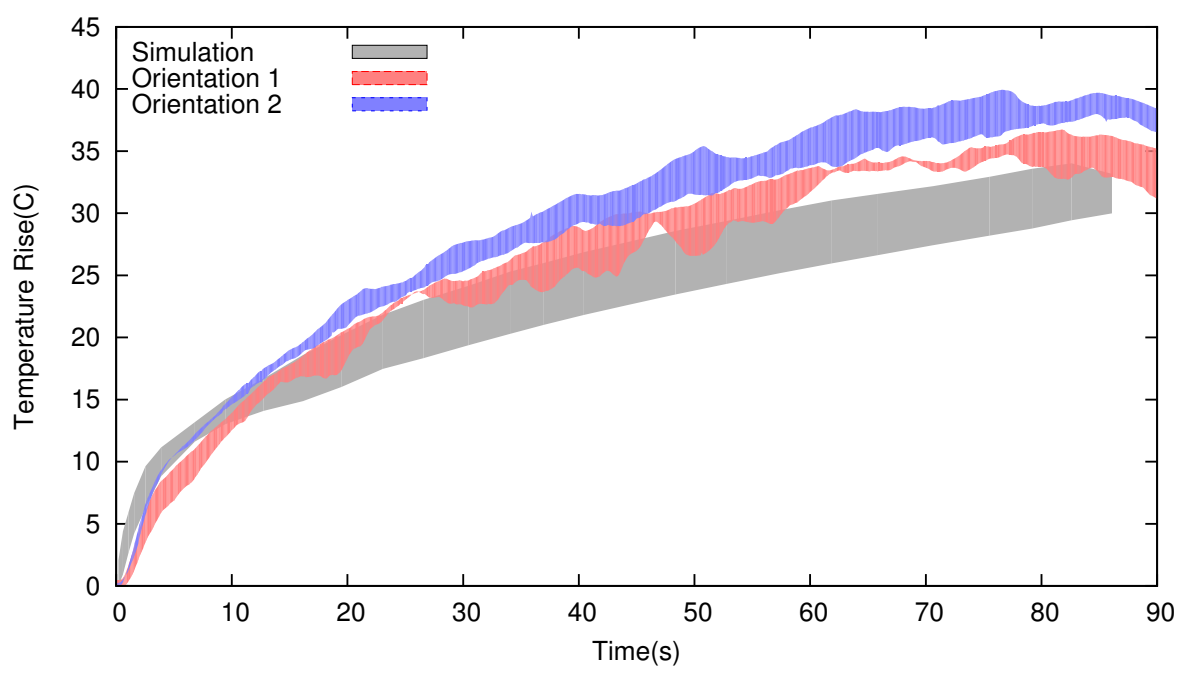

Figure 10: Comparisons between the experimental and simulation temperature rise at different location along tank centerline corresponding to location 1-5 in figure 2.

The five centerline thermo couples used to measure the instantaneous gas temperatures were used to generate the banded results. We can see that there is a slight difference between orientation 1 and 2. In the first few seconds, the experimental measurements lag the predicted temperature rise which is likely due to the thermocouple response time. After approximately 10 seconds the experiment shows a higher temperature rise than the prediction. 
This discrepancy increases to about a maximum of approximately $5^{\circ} \mathrm{C}$ by the end of the fill.

Figure 11 shows the measured and predicted temperature rise bands based on thermocouple locations 6-9 for both orientations. These were for thermocouples located on the liner surface inside the tank. Here, we can see that the bands are larger for orientation 2 than for 1 . This suggests that the buoyancy effects are causing larger temperature gradients between the thermocouples located at the 6 o'clock and 3 o'clock positions than 3 and 12 o'clock.

The predicted temperatures for these locations were, on average, in good agreement with the measurements but didn't show nearly as large a spread in top and bottom temperatures. Nevertheless, the results suggest that the liner material at the top of the tank is likely to experience the highest temperatures during service and may exceed the mass-averaged hydrogen temperature.

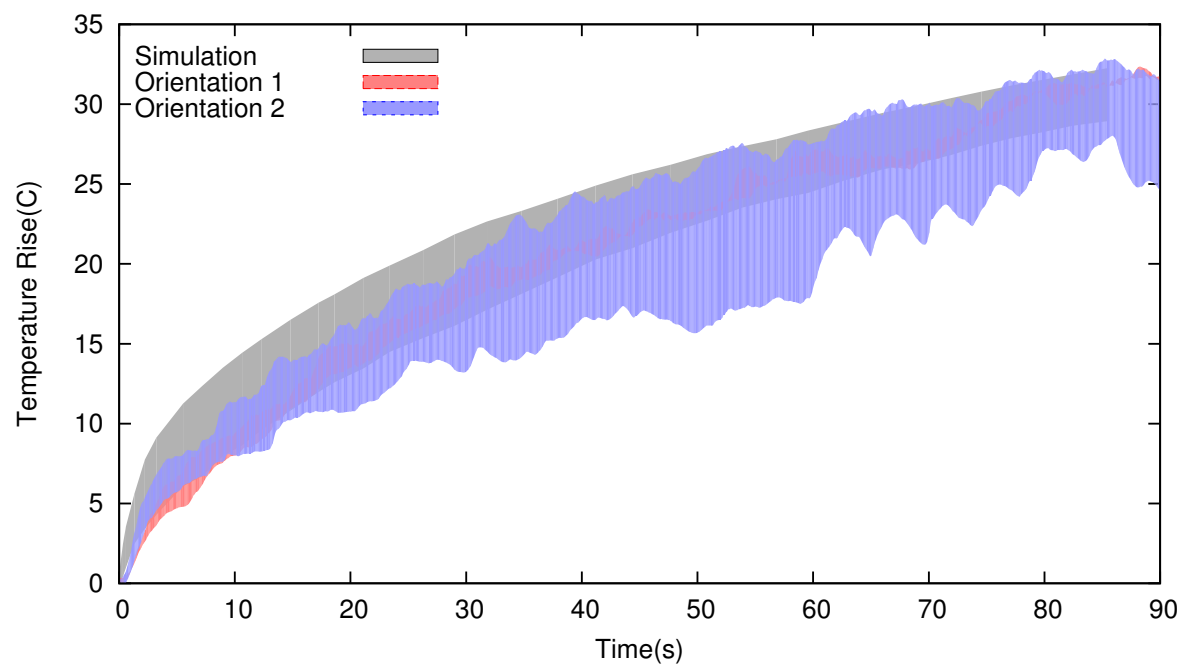

Figure 11: Comparisons between the experimental liner temperature rise and the simulation liner temperature rise. 
Figure 12 shows the measured and predicted temperature rise bands at the inner wall of the composite tank. This is the contact surface between the liner and the CFRP layer.

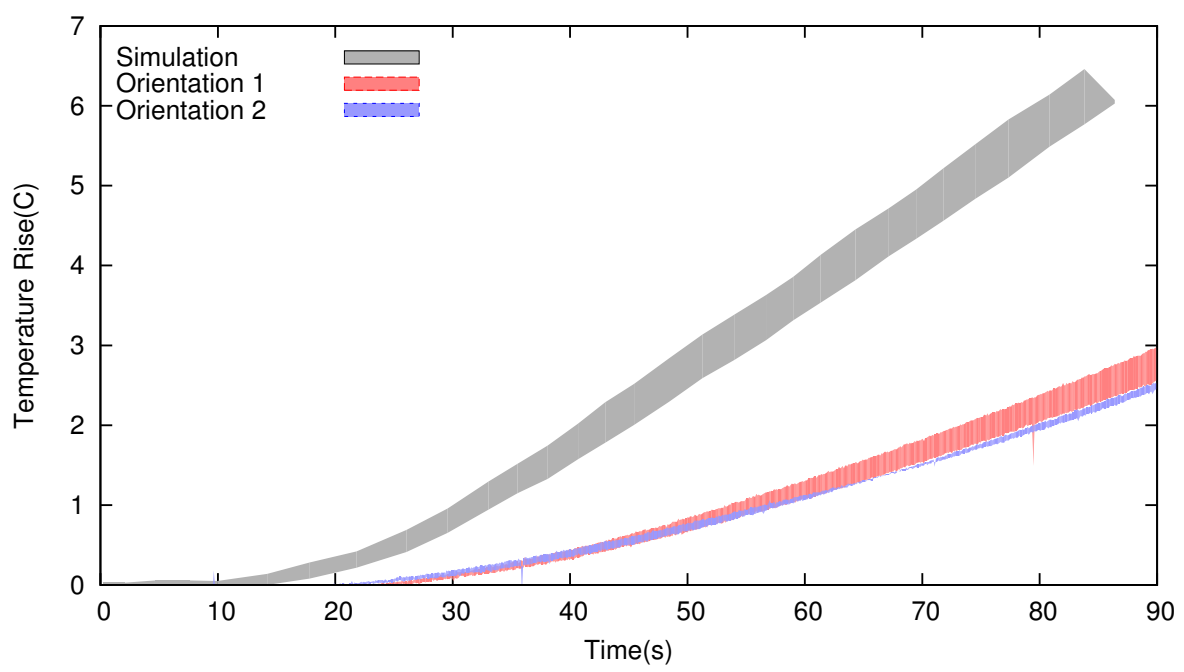

Figure 12: Comparisons between the experimental temperature rise and the simulation temperature rise of the inner wall.

The thermocouples are similarly distributed as those in the liner surface. However, in contrast to the liner thermocouples, which were epoxied directly to the liner, the inner wall thermocouples were inserted into drilled holes from the outside. An attempt was made to position them as near the liner-CFRP interface as possible without damaging the liner. Thus, it is likely that a thin layer of CFRP exists between these thermocouples and the liner. The figure shows that the 3D simulation over-predict the measured temperature rise by a significant amount. This could be caused by the difference in location of the thermocouples as well as the contact resistance between the liner and CFRP which was not explicitly modeled. Nonetheless, the measured and predicted 
results both show a low temperature rise during the duration of the fill. This is due to the low thermal conductivity of the HDPE liner which results in poor heat transfer to the composite layer. Both the experimental and simulation results had the highest temperature rise at the top center location. This result is consistent with the observed gas and liner temperature rise.

\section{Summary and Conclusion}

Experiments were carried out with a 39L Type IV tank and compared to one and three-dimensional models for validation. It has been demonstrated that good agreement between model predictions and experiment can be obtained for mass-averaged gas temperatures, mass inventory, and vessel pressure. However, due to the lumped nature of one-dimensional simulation codes, peak liner temperatures cannot be predicted. For the assessment of safety and to determine potential failure points, these peak temperature are required and a multi-dimensional procedure is necessary.

It was also shown through both experiment and simulation that the fast filling of high pressure hydrogen gas in Type IV tanks can produce temperatures in excess of $85^{\circ} \mathrm{C}$ if the fill procedure for Case 1 is followed. Case 2 was similar to case 1 but had a more moderate fill rate over a longer fill time. This resulted in a lower maximum gas temperature and a greater delivery of hydrogen gas. Case 3 also had similar fill conditions as cases 1 and 2 , however, the fill process was broken up into three 30 second steps. The first 60 seconds produced similar mass-flow rates as case 1 but because the fill process broken up over three steps, a lower maximum gas temperature was measured. This temperature was still greater than case 2 with a slightly 
lower mass delivery. Cases 4 and 5 had higher tank initial pressures and the lowest maximum gas temperatures. Because of lower compressibility effects, they also had the highest final mass storage. Thermal gradients in the wall temperatures were also shown to be influenced by buoyancy effects and tank materials.

This work showed that one-dimensional methods can be used for mass delivery and mass-averaged gas temperature predictions. However, due to buoyancy effects, the safety assessment of Type IV high-pressure tanks and the like will require high-fidelity three-dimensional modeling.

\section{Acknowledgments}

The authors wish to acknowledge Sandia National Laboratories. Sandia National Laboratories is a multi-program laboratory managed and operated by Sandia Corporation, a wholly owned subsidiary of Lockheed Martin Corporation, for the U.S. Department of Energys National Nuclear Security Administration under contract DE-AC04-94AL85000.

The authors would also like to thank Bill Winters for his help and consultation with the one-dimensional analysis.

\section{References}

[1] P. P. Edwards, Towards a sustainable energy future., Tech. Rep. 12.

[2] B. Sorensen, A. H. Petersen, C. Juhl, H. Ravn, Hydrogen as an energy carrier: scenarios for future use of hydrogen in the Danish energy system., International Journal of Hydrogen Energy 29 (1) (2004) 23-32. 
[3] S. Frankzky, High pressure 825 bar hydrogen storage. (2002).

[4] J. Y. Zheng, X. X. Liu, P. F. Liu, Y. Z. Zhao, J. Yang, Development of high pressure gaseous hydrogen storage technologies., International Journal of Hydrogen energy 37 (1) (2012) 1048-1057.

[5] J. Y. Zheng, J. J. Ye, J. Yang, An optimized control method for a high utilization ratio and fast filling speed in hydrogen refueling stations., International Journal of Hydrogen Energy 35 (7) (2010) 3011-3017.

[6] D. Mori, K. Hirose, Recentchallenges of hydrogenstorage technologies for fuel cell vehicles., International Journal of Hydrogen Energy 34 (10) (2009) 4569-4574.

[7] D. Chapelle, D. Perreux, Optimal design of a type 3 hydrogen vessel: part I-analytic modelling of the cylindrical section., International Journal of Hydrogen Energy 31 (5) (2006) 627-638.

[8] M. Monde, M. Woodfield, T. Takano, M. Kosaka, Estimation of temperature change in practical hydrogen pressure tanks being filled at high pressures of 35 and $70 \mathrm{MPa}$., International Journal of Hydrogen Energy 37 (7) (2012) 5723-5734.

[9] Gaseous hydrogen and hydrogen blends - Land vehicle fuel tanks., Tech. Rep. ISO/TS 15869, International Standard Organization (2009).

[10] W. S. Winters, Implementation and Validation of the NETFLOW Porous Media Model, Tech. rep., Sandia National Laboratories (2009). 
[11] M. Monde, Y. Mitsutake, A new estimation method of thermal diffusivity using analytical inverse solution for one-dimensional heat conduction, International Journal of Heat and Mass Transfer 44 (2001) 3169-3177.

[12] C. J. B. Dicken, W. Merida, Measured effects of filling time and initial mass on the temperature distribution within a hydrogen cylinder during refuelling., Journal of Power Sources 165 (1) (2007) 324-336.

[13] M. Heitsch, D. Baraldi, P. Moretto, Numerical investigations on the fast filling of hydrogen tanks., International Journal of Hydrogen Energy 36 (3) (2011) 2606-2612.

[14] A. Suryan, H. D. Kim, T. Setoguchi, Three dimensional numerical computations on the fast filling of a hydrogen tank under different conditions., International Journal of Hydrogen Energy 37 (9) (2012) 76007611.

[15] S. C. Kim, S. H. Lee, K. B. Yoon, Thermal characteristics during hydrogen fueling process of type IV cylinder., International Journal of Hydrogen Energy 35 (13) (2010) 6830-6835.

[16] M. C. Galassi, D. Baraldi, B. A. Iborra, P. Moretto, CFD analysis of fast filling scenarios for $70 \mathrm{MPa}$ hydrogen type IV tanks., International Journal of Hydrogen Energy 37 (8) (2012) 6886-6892.

[17] W. S. Winters, G. H. Evans, S. F. Rice, R. Greif, An experimental and theoretical study of heat and mass transfer during the venting of gas from pressure vessels., International Journal of Heat and Mass Transfer 55 (2012) 8-18. 
[18] D. R. Chenoweth, Gas-Transfer Analysis Section H- Real Gas Results via the van der Waals Equations of State and Virial Expansion Extension of its Limiting Abel-Noble form, Tech. Rep. SAND83-8229, Sandia National Laboratories (1983).

[19] J. T. Lyons, Heat transfer considerations in a pressure vessel being charged, Master's thesis (1969).

[20] J. D. Means, R. D. Ulrich, Transient convective heat transfer during and after gas injection into containers, Journal of Heat Transfer (1975) 282-287.

[21] G. L. Clark, A Zero-Dimensional Model of Compressible Gas Flow in Networks of Pressure Vessels-Program TRIC, Tech. Rep. SAND83-8226, Sandia National Laboratories (1983).

[22] C. N. Ranong, S. Maus, J. Hapke, G. Fieg, D. Wenger, Approach for the determination of heat transfer coefficients for filling processes of pressure vessels with compressed gaseous media, Heat Transfer Engineering 32 (2) (2011) 127-132.

[23] B. A. Younglove, M. O. McLinden, An International Standard Equation of State for the Thermodynamic Properties of Refrigerant 123 (2,2Dichloro-1,1,1-Trifluoroethane)., Journal of Physical and Chemical Reference Data 23 (5) (1994) 731-779. 